\title{
International Outcomes of COVID-19 during Pregnancy and a Literature Review on Maternal and Neonatal Outcomes
}

\author{
Sarwat Umer1, Maxine Reindorf', Rabia Zill-e-Huma1, Alex Wilkinson² \\ ${ }^{1}$ Department of Obstetrics and Gynaecology, East and North Hertfordshire NHS Trust, Stevenage, UK \\ ${ }^{2}$ Department of Respiratory Medicine, East and North Hertfordshire NHS Trust, Stevenage, UK \\ Email: saru_doc@hotmail.com
}

How to cite this paper: Umer, S., Reindorf, M., Zill-e-Huma, R. and Wilkinson, A. (2021) International Outcomes of COVID-19 during Pregnancy and a Literature Review on Maternal and Neonatal Outcomes. Open Journal of Obstetrics and Gynecology, 11, 450-460.

https://doi.org/10.4236/ojog.2021.114043

Received: March 20, 2021

Accepted: April 24, 2021

Published: April 27, 2021

Copyright $\odot 2021$ by author(s) and Scientific Research Publishing Inc. This work is licensed under the Creative Commons Attribution International License (CC BY 4.0).

http://creativecommons.org/licenses/by/4.0/

\begin{abstract}
Since the first report of COVID-19 in December 2019, our knowledge and treatment modalities have evolved at great pace understanding its impact on the maternal and neonatal outcomes. Whilst pregnant patients are not more susceptible to acquiring the infection as compared to the general population, they may be at higher risk of developing severe symptoms given the physiological changes, relative immune-compromised state of pregnancy, and their neonates at risk of consequent iatrogenic preterm deliveries. Our review article discusses outcomes of pregnant women and neonates with COVID-19 from several countries including the UK, USA, France, Italy and China and provides a narrative review of literature from November 2019 till December 2020.
\end{abstract}

\section{Keywords}

COVID-19, Pregnancy, Pregnancy Outcomes, SARS-CoV-2, Maternal Outcomes, Neonatal Outcomes

\section{Introduction}

Coronavirus disease 2019 (COVID-19) has evolved as a global pandemic since it was first reported in November 2019, with an estimated 94 million people infected and over 2 million deaths worldwide as of mid-Jan 2021 [1]. Whilst pregnant patients are not more susceptible to acquiring the infection in comparison to the general population [2], they may be at higher risk of developing severe symptoms given the relative immune-compromised state of pregnancy [3]. Furthermore, it poses a host of difficulties in the management, due to a number of 
physiological changes of pregnancy [4] and risk of iatrogenic preterm births [5] [6]. Skin to skin and breastfeeding widely recognised as highly beneficial in the post-partum period, however in some cases, mothers and babies have been separated following the birth in order to prevent possible transmission of the virus to the baby, without sufficient evidence to support this [7] [8].

Pregnant women, as a patient population, benefit a great deal from clinical research and trials. Because of lack of active inclusion of this group including in vaccinations trials, obstetricians and organisations have to rely on data from case series, case reports, observational and retrospective studies in order to inform guidance and clinical decision making.

The aim of this literature review is to establish the outcomes of pregnant patients and neonates with COVID-19 from some of countries heavily affected by the pandemic including the UK, USA, France, Italy and China published between from November 2019 to December 2020.

\section{Materials and Methods}

This is a narrative review of the literature published between November 2019 and December 2020. In July 2020, we carried out a literature search across PUBMED using the combined words "COVID-19", "pregnancy", "pregnancy outcomes", "SARS-CoV-2" and "neonatal outcomes". PECO framework was used for the review. The inclusion criteria included studies with original data for COVID-19 in pregnancy (suspected or confirmed) at any gestation of pregnancy (mean gestational age between 6 to 41 weeks) and neonates born to these mothers with COVID-19 in these studies. The exclusion criteria were postnatal and non-pregnant women. We excluded all the articles which were not in English.

Data was amalgamated into "regional" datasets. The data was collected using Microsoft Excel. Primary outcomes included maternal hospital and ITU admissions, maternal morbidity and mortality related to COVID-19 in pregnancy, risk of vertical transmission, neonatal morbidity and mortality related to COVID-19 in pregnancy. The secondary outcomes included effect of ethnicity on outcomes and impact on mode of delivery related to COVID-19 in pregnancy.

\section{Results}

The search yielded a total of 363 studies published during this time. Each paper was individually assessed and only those which presented original data were included. Total of 45 papers were finalised to review, and these were further categorised according to their country of origin.

Total 1211 suspected plus confirmed cases were identified with mean gestational age between 6 to 41 weeks. Out of these 648 (54\%) were from China, 428 (35\%) from Europe and 135 (11\%) were reported from USA and Canada (Table 1) 132 (11\%) women had co-morbidities; most commonly hypertension, diabetes mellitus, asthma and haematological diseases. Diabetes Mellitus was most frequently reported, affecting 63 (48\%) in European studies. 
Table 1. Summary of literature search.

Total studies: 363

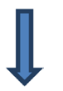

Included: 45 articles

- From UK, USA, France, Italy and China

- Articles in English language Excluded: 318

- Repetitive articles

- Non-English articles

Total cases: 1211

(Gestational age between 6 - 41 weeks)

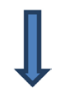

China: 648

Europe: 428

USA and Canada: 135

\subsection{Maternal Morbidity and Mortality}

Out of 1211 women, 82 (7\%) had Intensive care unit (ITU) admissions and 8 (0.7\%) required extracorporeal membrane oxygenation (ECMO). The highest number of ITU admission was noticed in the European studies, i.e. 41/428 (10\%). $55(4.5 \%)$ cases required invasive ventilation and $8(0.7 \%)$ received non-invasive ventilation. 7 (0.6\%) deaths had been reported, 2 from USA and 5 from the UK. The overall mortality rate among the patients admitted in ITU was $7 / 82$ (8.5\%).

$388(32 \%)$ of the women received antiviral or antibacterial agents. Highest numbers of antibacterial or antiviral agents were used in the Chinese studies 369/648 (57\%). Antenatal steroids were used in 117 (10\%) (Table 2).

\subsection{Mode of Delivery}

Completed birth data was available for 946 women. 14 women suffered miscarriage in this cohort (1.5\%). Live births were reported in 926/932 (99.4\%) cases and stillbirths in $6 / 932(0.6 \%)$ cases. None of miscarriages or stillbirths were related to COVID-19.

Out of 932 cases, 776 (83\%) underwent Caesarean Section (CS) whilst 156 (17\%) had vaginal delivery (Figure 1). On further analysis of CS, 324 (42\%) were done for conditions related to COVID-19 and 452 (58\%) for some other indication, previous CS being the most common. Overall CS rate was highest in China i.e., $92 \%$. Preterm birth rate was $21.5 \%$ (201/932). Most were iatrogenic preterm births 118/201 (58\%) and 85/201 (42\%) were spontaneous preterm births. 
Table 2. Maternal morbidity and mortality outcomes.

\begin{tabular}{|c|c|}
\hline Maternal outcomes & $\mathrm{N}(\%)$ \\
\hline Total women & 1211 \\
\hline ITU admissions & $82(7 \%)$ \\
\hline ECMO & $8(0.7 \%)$ \\
\hline Invasive ventilation & $55(4.5 \%)$ \\
\hline Non-invasive ventilation & $8(0.7 \%)$ \\
\hline Antiviral or antibacterial agents & $388(32 \%)$ \\
\hline Antenatal steroids & $117(10 \%)$ \\
\hline Maternal Deaths & $7(0.6 \%)$ \\
\hline Mortality rate among ITU admissions & $7(8.5 \%)$ \\
\hline
\end{tabular}

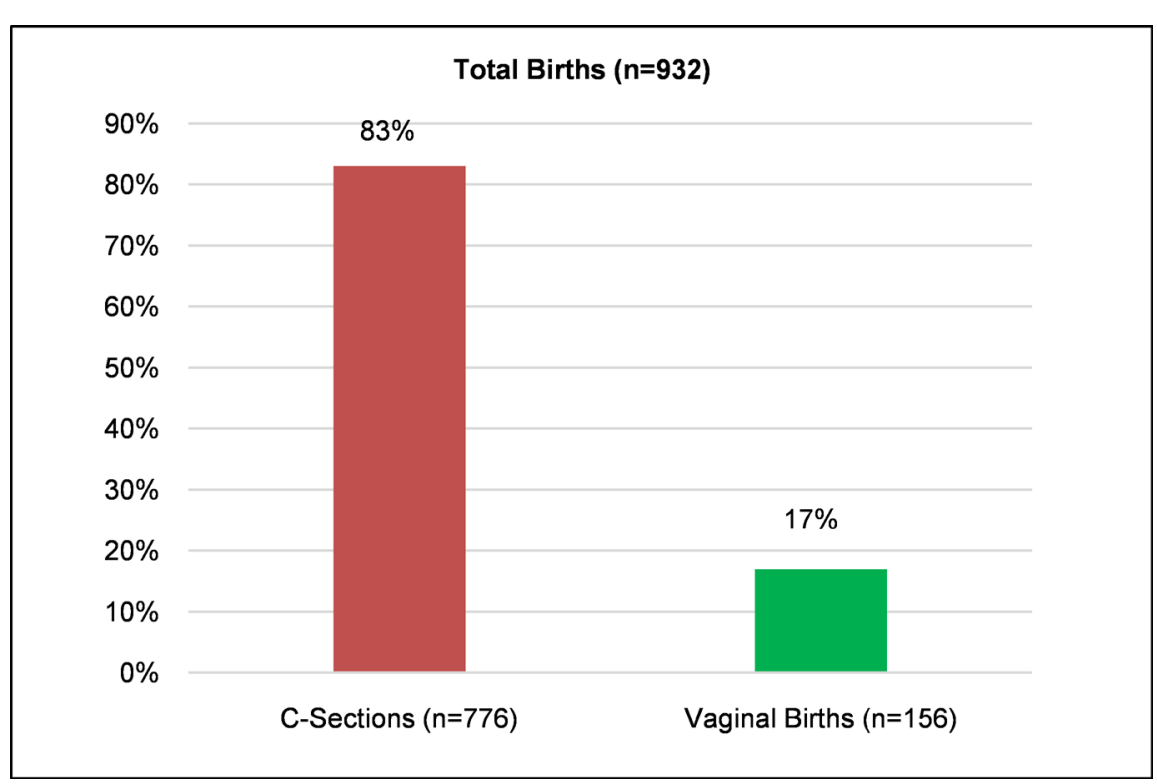

Figure 1. Mode of delivery.

\subsection{Neonatal Outcomes}

Completed birth data was available for 946 cases as mentioned earlier. Among 14 cases who suffered miscarriage, 9/14 (64\%) were reported from China and 6/9 were induced miscarriages because of concerns regarding unknown effect of COVID-19 on fetus. However, rests were spontaneous miscarriages.

COVID swabs were reported in 893 neonates. 14 (1.6\%) babies were positive for COVID-19<12 hours of birth. 11 (1\%) babies showed positive COVID-19 swabs $>12$ hours of birth. Majority (91\%) were tested negative (Figure 2). There were $12 / 926$ (1.3\%) neonatal deaths but none of them were related to COVID-19. There were no congenital anomalies noted. 


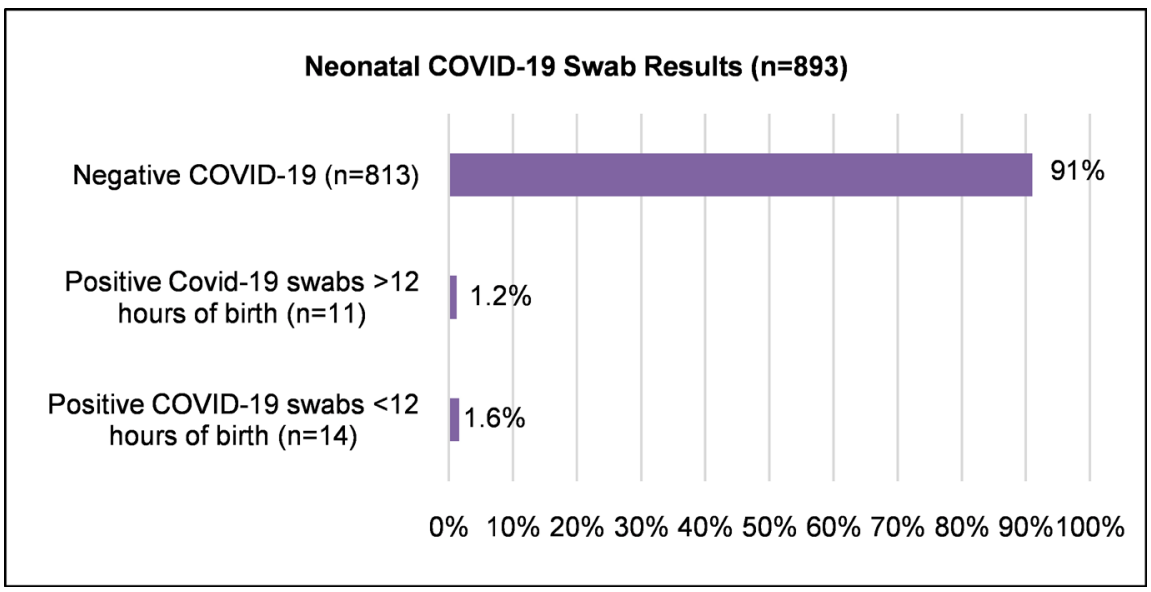

Figure 2. Neonatal COVID-19 swab results.

\section{Discussion}

Following the outbreak of COVID-19 in December 2019, there had been uncertainties regarding its impact on the pregnancy and neonatal outcomes. It was initially thought that pregnant women may be more susceptible to severe COVID-19 disease as compared to the general population as observed in MERS, SARS-COV-1 or ZIKA virus. Contrary to this, almost all the studies showed the risk of severe disease is not increased in pregnant women with SARS-COV-2 as compared to general population [9].

Looking at primary outcomes from maternal aspect in our review, $82(7 \%)$ had Intensive care unit (ITU) admissions and $8(0.7 \%)$ required extracorporeal membrane oxygenation (ECMO). Overall, 7 (0.6\%) deaths had been reported, 2 from USA and 5 from the UK. The overall mortality rate among the patients admitted in ITU was $7 / 82$ (8.5\%).11\% of women in our series had co-morbidities with diabetes being most common risk factor ( $48 \%$ in European studies).

Reviewing maternal morbidity and mortality in other studies, based on disease severity characteristics by $\mathrm{Wu}$ et al. [10] and comparison with pregnant population by Breslin et al. [11], 86\% exhibited mild disease, 9.3\% severe disease, $4.7 \%$ critical disease.

Allotey et al. [12] included 77 studies in their live systematic review on maternal and perinatal outcomes and found severe symptoms in 13\%, $4 \%$ admitted to ICU, $3 \%$ required ventilation, $0.4 \%$ received ECMO and $0.1 \%$ mortality rate.

Severe disease has been found to be associated with high BMI, vitamin D deficiency, multiple pregnancies, women with co-morbidities and in Black Asian and Minority ethnic group (BAME). Lokken et al. [13] identified 13\% (6/46) obstetric patients with severe disease had co-morbidities. United Kingdom Obstetric Surveillance System (UKOSS) reported 70\% of the pregnant women admitted to the hospital for COVID-19 were overweight or obese and a quarter of them had co-morbidities [14]. 56\% women were from Black Asian and Minority ethnic group (BAME) and were noted to be twice at risk of hospital admission and severe disease as compared to other ethnic groups. 
Overall maternal mortality with COVID-19 is $<1 \%$ as evident from our results, too and it corresponds with study by $\mathrm{N}$ Breslin et al. [11] $(<1 \%)$ as well as UKOSS (1\%). Maternal mortality in ICU admitted pregnant women is around $12 \%$; seen as $8 \%(7 / 82)$ in our review, $12.2 \%(5 / 41)$ in UKOSS, $15 \%(2 / 13)$ in Blitz et al. [15] and 12.9\% (11/85) in Kim et al. [16].

Looking at secondary outcomes of impact on mode of delivery in women with COVID-19 in pregnancy, we found that majority of women $(83 \%)$ underwent CS than vaginal delivery and it could be a reflection of different obstetric practices of mode of delivery in other countries rather than an obstetric clinical indication alone. For example, on further analysis of CS, $42 \%$ were done for conditions related to COVID-19 and 58\% for some other indication, previous CS being the most common. Overall CS rate was highest in China i.e., 92\%.

The rate of caesarean section (CS) had been reported in the range of $70 \%$ $100 \%$ in different other reviews as well. The most common indications included previous caesarean, foetal distress, meconium-stained liquor, co-morbidities and maternal COVID-19 infections [17]-[22]. The highest rate had been reported in China and the rates may differ in different countries on basis of their obstetric practice. Moreover, it could be related to concerns in early phases about risk of severe respiratory illness with COVID-19 as seen with other severe acute respiratory syndrome coronavirus (SARS-CoV) and Middle East respiratory syndrome coronavirus (MERS-CoV) infection with high maternal and foetal mortality. There was also concern about vertical transmission at vaginal delivery to the baby in early phases of pandemic. But the subsequent studies have shown that the vaginal secretions and amniotic fluid were negative for COVID-19, negating vertical transmission risk [18] [19] [20] [22] [23] [24] [25].

In our review, preterm birth rate was 21.5\% (201/932). Most were iatrogenic preterm births 118/201 (58\%) and 85/201 (42\%) were spontaneous preterm births. Any stress response in the body can cause preterm labour, but there is no direct association of COVID-19 observed with the preterm birth. Although there was increased risk of iatrogenic preterm delivery and caesarean section associated with moderate to severe infection in $3^{\text {rd }}$ trimester but mostly it was related to worsening maternal condition. However, preterm delivery and low birth weight was the single predictor for poor foetal outcome in women with COVID-19 [26].

There is no evidence of spontaneous miscarriage or any foetal abnormalities noted in infected women. Yang $\mathrm{Z}$ et al. and Yang $\mathrm{H}$ et al. reported that 2/114 and $4 / 27$ cases respectively opted for induced miscarriage earlier in pandemic due to unknown effect of COVID-19 on the foetus [27] [28].

Prabhu et al. [29] described placental histology in 28/30 (93\%) COVID-19 positive mothers and 99/305 (32.5\%) women without COVID-19. It was noticed that foetal vascular thrombosis $(\mathrm{p}<0.001)$ and meconium staining $(\mathrm{p}=0.004)$ were more common in COVID-19 positive patients. However, the frequency of chorioamnionitis was similar $(\mathrm{P}=0.0036)$. This might be explained by the increased risk of arterial and venous thromboembolism seen in COVID-19 pa- 
tients [30] [31].

Tutiya et al. reported 2 cases of severe COVID-19 infection that had thromboembolism on the $3^{\text {rd }}$ post caesarean day [32]. This might be because of combination of factors including sepsis, postnatal period, major pelvic surgery and immobility. It signifies that all pregnant and postnatal women with severe infection need to be assessed for higher doses of low molecular weight heparin than standard doses.

The risk of vertical transmission with COVID-19 is very low. Only $1.2 \%$ of babies in our review had positive COVID-19 swabs $>12$ hours after birth. None of the babies were unwell with COVID-19 related reasons. A recent systematic review by Walker et al. [8] evaluated possible routes of transmission of virus from mother to baby. Only 4.2\% (28/666) babies born to COVID-19 mothers tested positive and were all asymptomatic. None of them met the case definition of congenital COVID-19 infection, described by Shah et al. [33]. Hence, the true vertical transmission was not established. Risk of transmission was neither related to the mode of delivery nor was higher with breastfeeding and skin to skin contact. Currently, there is no policy to routinely test all neonates if born to asymptomatic or even mildly symptomatic mothers. Some countries don't have routine screening available to all labouring women. Nasopharyngeal and throat swabsimmediately after or within 12 hours of birth; risk false positive results due tomaternal secretions and IgM might represent maternal infection. Viral PCR in cord samples might have value but is not widely available. A few studies showed elevated IgG or IgM with negative PCR in new born babies [18] [30] [34]. The IgG are most likely maternally derived; however, IgM are larger molecules and cannot pass through placenta. It's postulated that the transfer of IgM can either be due to the placental damage caused by the infection or foetal IgM production secondary to in utero exposure of degenerated viral particles at foetal and placental interface. According to Wang et al., a decline in the neonatal IgG levels after 14 days represent maternal antibodies, but neonatal infection is more likely if the antibodies persist after 6 months [34].

Most of the studies reported mother and baby separation for 14 days to prevent horizontal transmission [22] [24] [30] [35] [36]. Walker et al., Breslin et al. and Nayak et al. showed that breast feeding and direct maternal contact does not affect the transmission to the baby, provided face mask and proper hand hygiene is practised [8] [11] [37].

Accumulating evidence supports ending infection control precautions using a symptom-based strategy. Concentrations of SARS-CoV-2 RNA decline after onset of symptoms, as does the likelihood of obtaining replication-competent virus, even when PCR tests remain positive. Patients with mild to moderate COVID-19 infection can be considered non-infectious 10 days after symptom onset. Persons with more severe to critical illness or severely immunocompromised likely remain infectious no longer than 20 days after symptom onset. Carefully explaining this to patients who may have had COVID-19 during pregnancy, particularly 
if recent test has been positive, may help reassure new mothers that they are no risk to their baby and thereby facilitate bonding and breastfeeding.

\section{Conclusions}

Our international review and analysis of literature show that pregnant women are not more susceptible to acquiring the infection in comparison to the general population. The risk of severe disease is not increased in pregnant women with SARS-COV-2 as compared to the general population. The risk of ITU admission in pregnant women with COVID-19 is usually $4 \%-7 \%$ with a need of invasive ventilatory support being $4 \%-5 \%$ and $0.4 \%-0.7 \%$ women requiring ECMO. Overall maternal mortality with COVID-19 is $<1 \%$. Maternal mortality in ICU admitted pregnant women is around $12 \%$.

Severe disease has been found to be associated with high BMI, vitamin D deficiency, multiple pregnancies, women with co-morbidities and in Black Asian and Minority ethnic group (BAME). Women with co-morbidities and BAME group should be advised to seek help earlier if their clinical condition deteriorates.

There is currently no evidence of spontaneous miscarriage or foetal abnormalities noted in women with COVID-19. Currently, there is no evidence of vertical transmission or spontaneous preterm delivery in these women as well. Foetal vascular thrombosis and meconium staining liquor have been reported in placental histology of some COVID-19 positive women but frequency of chorioamnionitis remains the same as general population risk. Mode and timing of delivery should be devised according to maternal medical condition in a multidisciplinary fashion with neonatologist, medical, anaesthetic and obstetric review. Women should be supported in breast feeding as there is no evidence about risk of neonatal transmission.

\section{Conflicts of Interest}

The authors declare no conflicts of interest regarding the publication of this paper.

\section{References}

[1] https://coronavirus.jhu.edu/map.html

[2] Royal College of Obstetricians and Gynaecologists and the Royal College of Midwives (2020) Coronavirus (COVID-19) Infection in Pregnancy: Information for Healthcare Professionals. Royal College of Obstetricians and Gynaecologists. https://www.rcog.org.uk/globalassets/documents/guidelines/2021-02-19-coronaviru s-covid-19-infection-in-pregnancy-v13.pdf

[3] Favre, G., Pomar, L., Musso, D. and Baud, D. (2020) 2019-nCoV Epidemic: What about Pregnancies? The Lancet, 395, e40. https://doi.org/10.1016/S0140-6736(20)30311-1

[4] LoMauro, A. and Aliverti, A. (2015) Respiratory Physiology of Pregnancy. Breathe, 11, 297-301. https://doi.org/10.1183/20734735.008615 
[5] Pierce-Williams, R.A.M., Burd, J., Felder, L., et al. (2020) Clinical Course of Severe and Critical COVID-19 in Hospitalized Pregnancies: A US Cohort Study. American Journal of Obstetrics \& Gynecology MFM, 2, 100134. https://doi.org/10.1016/j.ajogmf.2020.100134

[6] (2020) More on Clinical Characteristics of Pregnant Women with Covid-19 in Wuhan, China. The New England Journal of Medicine, 383, 696-697. https://doi.org/10.1056/NEJMc2016881

[7] Martins, V., Calil, L.T., Lucia, V., Krebs, J., Brunow De Carvalho, W. (2020) Guidance on Breastfeeding during the Covid-19 Pandemic. Revista da Associação Médica Brasileira, 66, 541-546. https://doi.org/10.1590/1806-9282.66.4.541

[8] Walker, K.F., O’Donoghue, K., Grace, N., Dorling, J., Comeau, J.L., Li, W. and Thornton, J.G. (2020) Maternal Transmission of SARS-Cov-2 to the Neonate, and Possible Route for Such Transmission: A Systematic Review and Critical Analysis. BJOG, 127, 1325-1336. https://doi.org/10.1111/1471-0528.16362

[9] Liu, Y., Chen, H., Tang, K. and Guo, Y. (2020) Clinical Manifestations and Outcome of SARS-CoV-2 Infection during Pregnancy. Journal of Infection. https://doi.org/10.1016/j.jinf.2020.02.028

[10] Wu, Z. and McGoogan, J.M. (2020) Characteristics of and Important Lessons from the Coronavirus Disease 2019 (COVID-19) Outbreak in China: Summary of a Report of 72,314 Cases from the Chinese Centre for Disease Control and Prevention. $J A M A, 323,1239-1242$. https://doi.org/10.1001/jama.2020.2648

[11] Breslin, N., Baptiste, C., Gyamfi-Bannerman, C., Miller, R., Martinez, R., Bernstein, K., Ring, L., Landau, R., Purisch, S., Friedman, A.M., Fuchs, K., Sutton, D., Andrikopoulou, M., Rupley, D., Sheen, J.J., Aubey, J., Zork, N., Moroz, L., Mourad, M., Wapner, R., Simpson, L.L., D’Alton, M.E. and Goffman, D. (2020) Coronavirus Disease 2019 Infection among Asymptomatic and Symptomatic Pregnant Women: Two Weeks of Confirmed Presentations to an Affiliated Pair of New York City Hospitals. American Journal of Obstetrics \& Gynecology MFM, 2, 100118.

https://doi.org/10.1016/j.ajogmf.2020.100118

[12] Allotey, J., Stallings, E., Bonet, M., et al. (2020) Clinical Manifestations, Risk Factors, and Maternal and Perinatal Outcomes of Coronavirus Disease 2019 in Pregnancy: Living Systematic Review and Meta-Analysis. BMJ, 370, m3320. https://doi.org/10.1136/bmj.m3320

[13] Lokken, E.M., Walker, C.L., Delaney, S., Kachikis, A., Kretzer, N.M., Erickson, A., Resnick, R., Vanderhoeven, J., Hwang, J.K., Barnhart, N., Rah, J., McCartney, S.A., Ma, K.K., Huebner, E.M., Thomas, C., Sheng, J.S., Paek, B.W., Retzlaff, K., Kline, C.R., Munson, J., Blain, M., LaCourse, S.M., Deutsch, G. and Adams Waldorf, K.M. (2020) Clinical Characteristics of 46 Pregnant Women with a Severe Acute Respiratory Syndrome Coronavirus 2 Infection in Washington State. American Journal of Obstetrics \& Gynecology, 223, 911.E1-911.E14. https://doi.org/10.1016/j.ajog.2020.05.031

[14] Knight, M., Bunch, K., Vousden, N., Morris, E., Simpson, N., Gale, C., O’Brien, P., Quigley, M., Brocklehurst, P. and Kurinczuk, J.J., UK Obstetric Surveillance System SARS-CoV-2 Infection in Pregnancy Collaborative Group (2020) Characteristics and Outcomes of Pregnant Women Admitted to Hospital with Confirmed SARS-CoV-2 Infection in UK: National Population-Based Cohort Study. BMJ, 369, m2107. https://doi.org/10.1136/bmj.m2107

[15] Blitz, M.J., Grünebaum, A., Tekbali, A., Bornstein, E., Rochelson, B., Nimaroff, M. and Chervenak, F. (2020) Intensive Care Unit Admissions for Pregnant and Non-Pregnant Women with Coronavirus Disease 2019. American Journal of $O b$ - 
stetrics \& Gynecology, 223, Article 6465.

[16] Kim, C.N.H., Hutcheon, J., van Schalkwyk, J. and Marquette, G. (2020) Maternal Outcomes of Pregnant Women Admitted to Intensive Care Units for Coronavirus Disease 2019. American Journal of Obstetrics \& Gynecology, 223, 773-774.

[17] Yan, J., Guo, J., Fan, C., Juan, J., Yu, X., Li, J., Feng, L., Li, C., Chen, H., Qiao, Y., Lei, D., Wang, C., Xiong, G., Xiao, F., He, W., Pang, Q., Hu, X., Wang, S., Chen, D., Zhang, Y., Poon, L.C. and Yang, H. (2020) Coronavirus Disease 2019 in Pregnant Women: A Report Based on 116 Cases. American Journal of Obstetrics \& Gynecology, 223, 111.E1-111.E14.https://doi.org/10.1016/j.ajog.2020.04.014

[18] Zimmermann, P. and Curtis, N. (2020) COVID-19 in Children, Pregnancy and Neonates: A Review of Epidemiologic and Clinical Features. The Pediatric Infectious Disease Journal, 39, 469-477. https://doi.org/10.1097/INF.0000000000002700

[19] Khan, S., Jun, L., Nawsherwan, Siddique, R., Li, Y., Han, G., Xue, M., Nabi, G. and Liu, J. (2020) Association of COVID-19 with Pregnancy Outcomes in Health-Care Workers and General Women. Clinical Microbiology and Infectious, 26, 788-790. https://doi.org/10.1016/j.cmi.2020.03.034

[20] Zhang, L., Jiang, Y., Wei, M., Cheng, B.H., Zhou, X.C., Li, J., Tian, J.H., Dong, L. and Hu, R.H. (2020) [Analysis of the Pregnancy Outcomes in Pregnant Women with COVID-19 in Hubei Province]. Chinese Journal of Obstetrics and Gynecology, 55, 166-171. (In Chinese)

[21] Yu, N., Li, W., Kang, Q., Xiong, Z., Wang, S., Lin, X., Liu, Y., Xiao, J., Liu, H., Deng, D., Chen, S., Zeng, W., Feng, L. and Wu, J. (2020) Clinical Features and Obstetric and Neonatal Outcomes of Pregnant Patients with COVID-19 in Wuhan, China: A Retrospective, Single-Centre, Descriptive Study. Lancet Infectious Diseases, 20, 559-564. https://doi.org/10.1016/S1473-3099(20)30176-6

[22] Chen, H., Guo, J., Wang, C., Luo, F., Yu, X., Zhang, W., Li, J., Zhao, D., Xu, D., Gong, Q., Liao, J., Yang, H., Hou, W. and Zhang, Y. (2020) Clinical Characteristics and Intrauterine Vertical Transmission Potential of COVID-19 Infection in Nine Pregnant Women: A Retrospective Review of Medical Records. The Lancet, 395, 809-815. https://doi.org/10.1016/S0140-6736(20)30360-3

[23] Panahi, L., Amiri, M. and Pouy, S. (2020) Risks of Novel Coronavirus Disease (COVID-19) in Pregnancy: A Narrative Review. Archives of Academic Emergency Medicine, 8, e34.

[24] Khan, S., Peng, L., Siddique, R., Nabi, G., Nawsherwan, Xue, M., Liu, J. and Han, G. (2020) Impact of COVID-19 Infection on Pregnancy Outcomes and the Risk of Maternal-to-Neonatal Intrapartum Transmission of COVID-19 during Natural Birth. Infection Control \& Hospital Epidemiology, 41, 748-750. https://doi.org/10.1017/ice.2020.84

[25] Peng, Z., Wang, J., Mo, Y., Duan, W., Xiang, G., Yi, M., Bao, L. and Shi, Y. (2020) Unlikely SARS-CoV-2 Vertical Transmission from Mother to Child: A Case Report. Journal of Infection and Public Health, 13, 818-820. https://doi.org/10.1016/j.jiph.2020.04.004

[26] Prabhu, M., Cagino, K., Matthews, K., Friedlender, R.L., Glynn, S.M., Kubiak, J.M., et al. (2020) Pregnancy and Postpartum Outcomes in a Universally Tested Population for SARS-COV-2 in New York City: A Prospective Cohort Study. BJOG, 127, 1548-1556. https://doi.org/10.1111/1471-0528.16403

[27] Cheruiyot, I., Kipkorir, V., Ngure, B., Misiani, M., Munguti, J. and Ogeng'o, J. (2020) Arterial Thrombosis in Coronavirus Disease 2019 Patients: A Rapid Systematic Review. Annals of Vascular Surgery, 70, 273-281. 
[28] Nopp, S., Moik, F., Jilma, B., Pabinger, I. and Ay, C. (2020) Risk of Venous Thromboembolism in Patients with COVID-19: A Systematic Review and Meta-Analysis. Research and Practice in Thrombosis and Haemostasis, 4, 1178-1191. https://doi.org/10.1002/rth2.12439

[29] Mehan, A., Venkatesh, A. and Girish, M. (2020) COVID-19 in Pregnancy: Risk of Adverse Neonatal Outcomes. Journal of Medical Virology, 92, 2295-2297. https://doi.org/10.1002/jmv.25959

[30] Yang, Z., Wang, M., Zhu, Z. and Liu, Y. (2020) Coronavirus Disease 2019 (COVID-19) and Pregnancy: A Systematic Review. The Journal of Maternal-Fetal \& Neonatal Medicine, 1-4. https://doi.org/10.1080/14767058.2020.1759541

[31] Yang, H., Hu, B., Zhan, S., Yang, L.Y. and Xiong, G. (2020) Effects of SARS-CoV-2 Infection on Pregnant Women and Their Infants: A Retrospective Study in Wuhan, China. Archives of Pathology \& Laboratory Medicine, 144, No. 10.

[32] Tutiya, C.T., Siaulys, M.M., Kondo, M.M., Miglioli-Galvão, L., Galvão, E.C.A., Pinheiro, C.C., Torloni, M.R. and de Mello, F.B. (2020) Possible Formation of Pulmonary Microthrombi in the Early Puerperium of Pregnant Women Critically Ill with COVID-19: Two Case Reports. Version 2. Case Reports in Women's Health, 27, e00237. https://doi.org/10.1016/j.crwh.2020.e00237

[33] Shah, P.S., Diambomba, Y., Acharya, G., Morris, S.K. and Bitnun, A. (2020) Classification System and Case Definition for SARS-CoV-2 Infection in Pregnant Women, Fetuses, and Neonates. Acta Obstetricia et Gynecologica Scandinavica, 99, 565-568. https://doi.org/10.1111/aogs.13870

[34] Wang, C., Zhou, Y.H., Yang, H.X. and Poon, L.C. (2020) Intrauterine Vertical Transmission of SARS-CoV-2: What We Know So Far. Ultrasound in Obstetrics \& Gynecology, 55, 724-725. https://doi.org/10.1002/uog.22045

[35] Masmejan, S., Pomar, L., Lepigeon, K., Favre, G., Baud, D. and Rieder, W. (2020) COVID-19 et Grossesse [COVID-19 and Pregnancy]. Revue Médicale Suisse, 16, 944-946.

[36] Liu, W., Wang, J., Li, W., Zhou, Z., Liu, S. and Rong, Z. (2020) Clinical Characteristics of 19 Neonates Born to Mothers with COVID-19. Frontiers of Medicine, 14, 193-198. https://doi.org/10.1007/s11684-020-0772-y

[37] Nayak, A.H., Kapote, D.S., Fonesca, M., Chavan, N., Mayenkar, R., Sarmalkar, M. and Bawa, A. (2020) Impact of Coronavirus Infection in Pregnancy: A Preliminary Study of 141 Patients. The Journal of Obstetrics and Gynecology of India, 70, 256-261. https://doi.org/10.1007/s13224-020-01335-3 